\title{
OCCSR: Document Classification by Order of Context, Concept and Semantic Relations
}

\author{
A. Venkata Ramana* and E. Kesavulu Reddy \\ Department of Computer Science, \\ S. V. University, Tirupati - 517502, Andhra Pradesh, India; \\ ekreddysvu2008@gmail.com,avr_rdg@yahoo.co.in
}

\begin{abstract}
The contemporary research in text or document mining is discerning towards syntactic components and semantic environment. In order to this and with the motivation gained from our earlier research contributions, here we explored a mining model to classify documents by Order of Context, Concept and Semantic Relations (OCCSR). This proposed model is classifying the documents in three levels and they are by context, by concept and semantic. The document context is defined through the document meta-data, the concept is defined by the order of features and semantic relations are assessed through the correlation off the activities observed in documents. The experimental results explored are indicating that the OCCSR is with high classification accuracy, scalable and robust. The research findings lead us to conclude that the context similarity along with concept and semantic similarity score is more significant to achieve classification accuracy in supervised learning. Assessment of the OCCSR is done by using confusion matrix and discriminator metrics. The model devised here is most useful, in particular to assess relation of the documents published in social communities like electronic journals, publishers and blogs.
\end{abstract}

Keywords: Concept Relations, Context Relations, Document Classification, Feature Selection, Semantic Relations, Supervised Learning, Text Mining, OCCSR

\section{Introduction}

Text mining efforts to explore newer, earlier not known data by employing methods from normal language process as well as data mining. Categorization, among conventional text mining strategies, is monitored learning perspective in which categorization techniques attempt to designate a doc to several classifications, according to the doc information. Classifiers are proficient from illustrations to perform the classification work instantly. A lot of recent report categorization techniques are according to the Vector Space Model (VSM) ${ }^{1-3}$ that is a commonly employed data description. The VSM signifies every doc as a characteristic vector of the jargon (words or phrases) in the doc. Every characteristic vector consists of term loads (usually term-frequencies) of the words in the doc. The resemblance in between documents is assessed by one of the resemblance actions that are according to that a characteristic vector. Illustrations consist of the cosine estimate as well as the Jaccard measure.

The similarity determines shows the point of distance or splitting of the desired objects and might represent to the aspects that are suspected to identify the clusters enclosed in the data. Earlier clustering, a similarity/distance assess should always be confirmed ${ }^{4}$. Selecting an applicable similarity step is also significant for cluster assessment, particularly for a specific kind of clustering algorithms.

${ }^{*}$ Author for correspondence 
Text Categorization (TC) is the categorization of information in regard to a collection of one or additional preexisting aspects ${ }^{5}$. The categorization phase includes of building a weighted vector for every aspect and then applying a resemblance assesses to find the nearest category. The resemblance determines is used to identify the level of similarity in between two vectors. To accomplish reasonable categorization results, a similarity evaluate must usually respond with significant values to information that should be to the similar class and with modest values commonly. All through the past decades, a huge number of techniques endorsed for text categorization had been commonly built on the traditional Bag-ofWords version where each and every term or term stem is a self-governing feature.

The prevailing similarity strategy was much more usually used to analyze the resemblance in between words. However the content theoretic likeness determines results are mathematically noticeable it does not diminish the specifications of the vector model ${ }^{6}$. Metric ranges such that Euclidean distance is not really suitable for high specifications and sparse fields. Owning to the situation of any regards in between words, the learning algorithms are forbidden to identify patterns in the included terminology only, although conceptual patterns persist dismissed.

Prevailing strategies requires doing an optimization more than a whole assortment of documents. Many of these strategies are computationally expensive.

The improvements in this field are accelerated by tough theoretical motivations. This is because of the machine learning methods in the text classification field. For this classification a good number of machine learning techniques are used, which include example-based classifiers, neural networks, Rocchio method, nearest neighbor classifiers, regression methods, decision trees and probabilistic classifiers ${ }^{7}$.

Vapnik introduced new learning methods Support Vector Machines (SVMs) in 1995,9. Promising results were got in later years when many studied made use of SVMs for text classification ${ }^{8-13}$. Joachims presented the primary studies that brought in SVMs for text classifications in 1998. The study shows the comparison of non-linear model with four popular machine learning algorithms Naïve Bayes (NB) classifier, Rocchio method, k-nearest neighbor (k-NN) classifier and C4.5 decision tree) on Reuters and Ohsumed datasets. We can conclude that SVM is perfect for test classification and most importantly is better than the other methods. Dumais, et al. in the same year checked the precision of five various machine learning algorithms on Reuters dataset for text classification and wrapped up with the result that the precision of the simple linear SVM is one of the best reported for Reuters alike the Joachims study. Linear SVM is specifically promising as it is much easy and more competent that Joachims non-linear model ${ }^{10}$. Yang and Liu conducted a controlled study with statistical important tests on five learning algorithms (SVM, k-NN, Neural Network (NNet) method, NB and Linear Least-Square Fit (LLSF) mapping). The conclusion was that SVM is one of the most successful machines to learn algorithms. A survey was present by Sebastiani which covered the main machine learning approaches in test classifications ${ }^{11}$.

Text classification has another major issue which is decreasing dimensionality. By using feature selection we can obtain precision and efficiency of classifiers by choosing more discriminative terms in datasetsasfeatures.Differentfeatureselection methodshave been shown and checked in literature ${ }^{14}$. Five various feature selection methods were analyzed by Yang and Peterson ${ }^{15}$, on Reuters and Ohsumed datasets by making use of k-NN and LLSF categorization algorithms in the case of global policy. IG and CHI methods are termed to be the most successful methods ${ }^{15}$. An empirical comparison was drawn by Forman. He compared twelve feature selection methods. This was done on a benchmark that was got from Reuters, TREC and Ohsumed, by making use of SVM in case of local policy. Outstanding performance was shown by accuracy and F-measure. Specifically on highly skew datasets yet it was IG that yielded the best results in exactness. Debole and Sebastiani in the same year suggested Supervised Term Weighting (STW) scheme by making use of IG, CHI and Gain Ratios (GR) along with TF-IDF weighting on Reuters dataset along with SVM in both local and global policies. It was finalized that the GR performs better than the other methods and gave exemplary results as a STW function specifically in macro-averaged F-measure ${ }^{16}$.

On Reuters data along with SVM in local and global policies Ozgur, et al. ${ }^{17}$ compared TF-IDF weighting with Boolean weighting. Compared to Boolean weighting it was observed that TF-IDF performed better. It was also seen that global policy performed better for a large number of keywords than small number of keywords. In such case local policy outperformed the global policy ${ }^{17}$. In a study thereafter Ozgur and Gungor checked the 
performance of these two policies along with two weightings and six other document collections. Added to this there were skewed properties along with various numbers of keywords using SVM in detail in 2006. Also it was proven that the results of the earlier studies can simplify that the global policy is a better performer when it comes to large number of keywords and local policy performs well for small number of keywords and in skew datasets ${ }^{14}$. Tasci and Gungor ${ }^{14}$, used the analysis with different existing feature selection methods and four other suggested feature selection methods that are similar to Acc2 metric. The feature selection methods on six standard document collections were compared by changeable number of selected feature from 10 to 2000 in both local and global policy. Also they came to a conclusion that Acc2 is the finest metric among the existing metric, that too with a limited number of features. The victory of Acc2 was clear in local policy on skew datasets.

Contrary to this the suggested metric M1 is more appealing that the victorious metric Acc2 in the experiments ${ }^{18,19}$.

Liu, et $\mathrm{al}^{20}$, centered on data imbalance issue in text classification by showing a probability based term weighing scheme that was stimulated by various feature selection approach. They wrapped up that making use of probability based term weighing scheme can perk up categorization performance on rare classes.

Different researches have been performed to better the working of feature selection approaches on text categorization. But these basically deal with the improvement of the performance of every single feature selection approach. Also, it is difficult to say which feature selection method is better than the other, even though there are many feature selection methods in text classification. In addition, the text is categorized based on the heaviness of the feature in relation to conceptualization but not in term relation. For example both coal mining and data mining can be put into one class called as mining, which is not accurate in the context of concept relation. Therefore in our earlier research article ${ }^{21}$ defined a measuring metric called feature relationship weights that help us describe the class labels not by terms but by concepts. Further the Semantic labeling was devised ${ }^{22}$ by us, which is an extension to the concept labeling. With the motivation gained from the experimental results of these two models, here we proposed a document classification strategy that classifies the documents in the order of context, concept and semantic relations.

\section{Document Classification by the Order of Context, Concept and Semantic Similarities}

The projected approach is classifying given text documents by the order of context, concept and semantic relations the first order classification is done by the context relations that are extracted from the metadata of the given documents and corpus. The second order of classification is to classify the documents by concept and further in third order, these documents will be categorized by their semantic relations that are extracted from the correlation of the activities and concepts. The metric used in first order to classify the given documents is context weight and the second order classification is categorizing the documents by concept weights, which is measured from the feature correlation ${ }^{21}$. Finally the third order of the projected model classifies these documents by the correlation of the activities found in the given text document. The second order classification of the proposed model is using the verbs as activities and arguments as concepts.

\subsection{Data Preprocessing}

The initial step of the projected model is to preprocess the text data of given documents dataset. At first the text data will be tokenized and then the stop words will be removed. Further the leftover words will be processed by stemming, which mainly to remove tenses. Further these tokens are bipartite as activities (the verbs) and concepts (the arguments).

\subsection{First Order Classification of Documents by Metadata as Context}

Initially we extract the authors, document published date and keywords of the given documents as metadata. Then the total time range of the documents publishing dates in ascending chronological order will be partitioned into set of time zones, which is based on given time zone threshold $m t z$. Further the keywords and authors will be grouped according to the documents published in respective time zones. In detail, the authors of the documents those published in a time zone will be considered as one group and similarly the keywords of the documents published in single time zone also be considered as one group.

These time zones, author groups and keyword groups are used to classify the documents in first order. In regard to this a heuristic scale will be developed to confirm the 
class of a specific document. The algorithmic exploration of first order classification is following.

- Initially Form the set of Time Zones $T Z=\left\{t z_{1}, t z_{2}, t z_{3}, \ldots t z_{n}\right\}$, set of author groups $A G=\left\{a g_{1}, a g_{2}, \ldots \ldots, a g_{n}\right\}$ and set of keyword groups $K W G=\left\{k w g_{1}, k w g_{2} \ldots \ldots k w g_{n}\right\}$

- For each document $d_{i}$ of the given corpus, the metadata will be extracted.

- The all of the dates involved in document creation publishing and versioning will be grouped as $v d g\left(d_{i}\right)=\left\{v d_{1}, v d_{2}, \ldots \ldots v d_{\mid v d g\left(d_{i}\right)}\right\}$

- The authors of the document will be considered as a group $\operatorname{dag}\left(d_{i}\right)=\left\{a_{1}, a_{2}, \ldots \ldots a_{\left|\operatorname{dag}\left(d_{i}\right)\right|}\right\}$

- And the index terms or keywords representing that document will be grouped as $\operatorname{itg}\left(d_{i}\right)=\left\{k w_{1}, k w_{2}, \ldots . . k w_{\mid \operatorname{ltg}\left(d_{i}\right)}\right\}$.

- Further bipartite graphs will be formed between

- attributes of $T Z$ and attributes of $\operatorname{vdg}\left(d_{i}\right)$ (Figure 1),

- attributes of $A G$ and attributes of $\operatorname{dag}\left(d_{i}\right)$ (Figure 2),

- attributes of $K W G$ and attributes of (Figure 3)

- Further the edges of these bipartite graph will be weighted by the ratio of the similarity

- Further similarity score of the document with TZ, AG and $K W G$ will be assessed by the Likert scale strategy.

- Then the documents will be labeled as a class $\left\{t z_{i}, a g_{i}, k w g_{i}\right\}$ if and only if the similarity score between given document and class $\left\{t z_{i}, a g_{i}, k w g_{i}\right\}$ is maximum that compared to the similarity scores of the given document with other class labels.

\subsubsection{Pseudo Representation of the First Order Classification of the Documents by Context}

\subsubsection{Preprocessing}

- Let $D C=\left\{d_{1}, d_{2}, \ldots \ldots . . d_{|D C|}\right\}$ be the given documents of size $|D C|$ with metadata

- Find the document versioning time frame between the date and time of first published document to most recent published document as $T Z$.

- Partition $T Z$ as equal time zones $\left\{t z_{1}, t z_{2}, \ldots . ., t z_{|T Z|}\right\}$ of size $|T Z|$ by given threshold $t t$

- Extract all the authors as a set $A L$ with no duplicates of all documents of corpus $D C$

- Create set of author groups $A G=\left\{a g_{1}, a g_{2}, \ldots \ldots . ., a g_{n}\right\}$ such that each group $\left\{a g_{i} \exists a g_{i} \subset A G\right\}$ contains the authors involved in documents of each time zone $\left\{t z_{i} \exists t z_{i} \subset T Z\right\}$

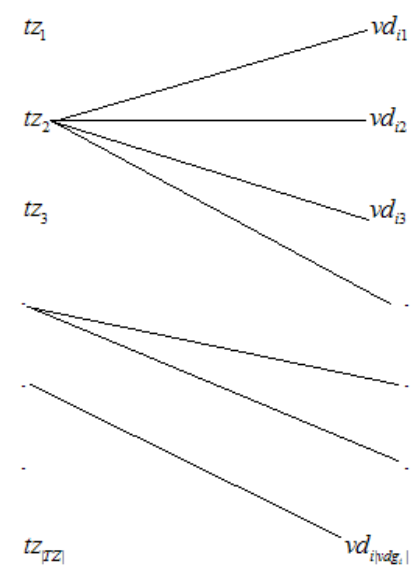

Figure 1. Bipartite graph between time zones and $\mathrm{i}^{\text {th }}$ document versioning dates.

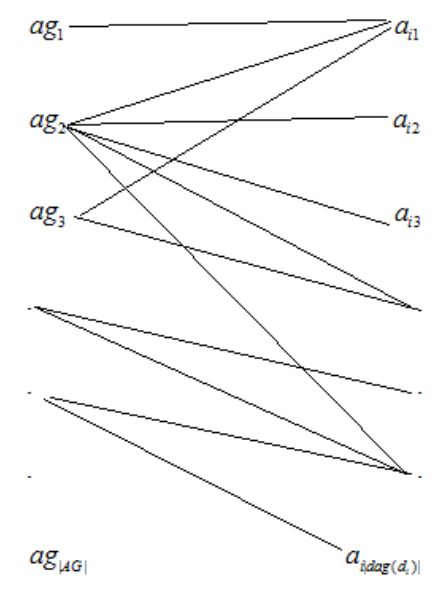

Figure 2. Bipartite graph between author groups and authors of $\mathrm{i}^{\text {th }}$ document.

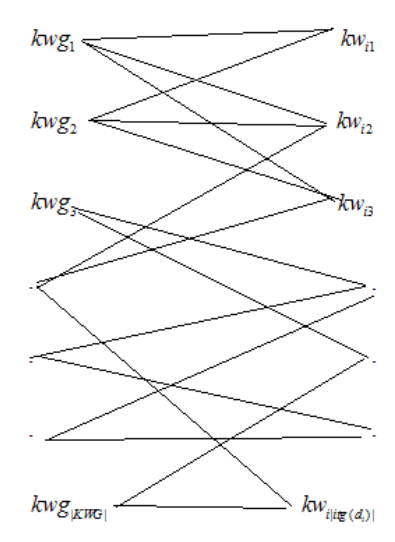

Figure 3. Bipartite graph between keyword groups and keywords of $\mathrm{i}^{\text {th }}$ document. 
- Extract all the keywords as unique list $K W L$ that are part of metadata from the documents of the corpus DC

- Create set of keyword groups $K W G=\left\{k w g_{1}, k w g_{2}, \ldots \ldots\right.$ $\left.k w g_{n}\right\}$ such that each group $\left\{k w g_{i} \exists k w g_{i} \subset K W G\right\}$ contains the keywords used in documents of each time zone $\left\{t z_{i} \exists t z_{i} \subset T Z\right\}$

\subsubsection{Classification}

- $t f r \leftarrow \phi / / a$ set that contains the time zone ratios of documents

- $\operatorname{ar} \leftarrow \phi / /$ a set that contains the author ratios of documents

- $k w r \leftarrow \phi / / a$ set that contains the keyword ratios of the documents

- For each document $\left\{d_{i} \exists d_{i} \in D C \wedge i=1,2,3, \ldots .|D C|\right\}$ Begin

- Extract all versioning dates $v d g\left(d_{i}\right)=\left\{v d_{1}, v d_{2}, \ldots \ldots . . v d_{\left|v d g\left(d_{i}\right)\right|}\right\}$ of document $d_{i}$

- Extract all authors $\operatorname{dag}\left(d_{i}\right)=\left\{a_{1}, a_{2}, \ldots . . a_{\left|\operatorname{dag}\left(d_{i}\right)\right|}\right\}$ involved in versioning of document $d_{i}$

- Extract all keywords $i \operatorname{tg}\left(d_{i}\right)=\left\{k w_{1}, k w_{2}, \ldots . . k w_{\left|i t g\left(d_{i}\right)\right|}\right\}$ used in the metadata of the document $d_{i}$

- Let set $t f r\left(d_{i}\right) \leftarrow \phi / /$ set that contains time zone relation ratios

- For-each $\left\{t z_{j} \in T Z \exists j=1,2, \ldots .|T Z|\right\}$ Begin

- $t f r\left(d_{i}\right) \leftarrow \frac{\sum_{k=1}^{\left|v d g\left(d_{i}\right)\right|}\left\{1 \exists t z_{j-1}<v d_{k} \leq t z_{j}\right\}}{\left|v d g\left(d_{i}\right)\right|}$

- End

- Let set $\operatorname{ar}\left(d_{i}\right) \leftarrow \phi / /$ set of authors ratios

- For-each $\left\{a g_{j} \in A G \exists j=1,2, \ldots .|A G|\right\}$ Begin

- $\operatorname{ar}\left(d_{i}\right) \leftarrow \frac{\sum_{k=1}^{\left|\operatorname{dag}\left(d_{i}\right)\right|}\left\{1 \exists a_{k} \in a g_{j}\right\}}{\left|\operatorname{dag}\left(d_{i}\right)\right|}$

- End

- Let set $k w r\left(d_{i}\right) \leftarrow \phi / /$ set of keywords ratios

- For-each $\left\{k w g_{j} \in K W G \exists j=1,2, \ldots .|K W G|\right\}$ Begin

- $k w r\left(d_{i}\right) \leftarrow \frac{\sum_{k=1}^{\left|i \operatorname{tg}\left(d_{i}\right)\right|}\left\{1 \exists k w_{k} \in k w g_{j}\right\}}{\left|i \operatorname{tg}\left(d_{i}\right)\right|}$

- End

- Let $\operatorname{cs}\left(d_{i}\right) \leftarrow \phi$ //a set that contains class scores of document $d_{i}$

- For-each $\{k=1,2, \ldots . .[|t f r| \vee|a r| \vee|k w r|]\}$ Begin
- $k w r\left(d_{i}\right)_{[k]} * 100+\operatorname{ar}\left(d_{i}\right)_{[k]} * 75+t f r\left(d_{i}\right)_{[k]} * 50$

//applying Likert scale to assess the impact of class label, which is in the order of keywords, authors and time zone respectively

- End

- The class with highest class score $\left\{c s\left(d_{i}\right)_{[k]} \exists k \in\left[1,2,3, \ldots \ldots . .,\left|t f r\left(d_{i}\right)\right|\right\}\right.$ will be opted as class label of the document $d_{i}$

- End

\subsection{Second Order Classification by Concept Labeling 21}

The second order classification categorizes the documents of each first order class label by concept, which is based on our earlier work ${ }^{21}$. In regard to this, initially finds the concept weights and then the features are pruned through the metric called similarity score. The two approaches called measuring concept weights and pruning features by similarity score metric are briefed in following sections.

\subsubsection{Concept Weights by Feature Correlation}

The increasing order series of the features and their happening in the said set of documents is known as Concept Weight (CW). The ordered series is originally considered with lone feature and then it rises by adding every feature per iteration. The sequence is terminated once the Concept Weight is found to be less than the said threshold. In this process if the series s1 is subset of sequence s2 and concept weight of s1 is less than or equal to concept weight of $s 2$ then $s 1$ can be trimmed. This procedure results set of hypothesis as feature set 'CFS'

\subsubsection{Feature Pruning by Similarity Score}

Feature with many terms will be got in this phase and the obtain the parallel score in each selected feature $\mathrm{x}$ and other each feature $\mathrm{x}^{\prime}$ with lesser number of terms than $\mathrm{x}$. in case the semblance between $\mathrm{x}$ and $\mathrm{x} 1$ are found to be more than the said threshold and bigger than all of the similarity scores between $x^{\prime}$ and rest of bigger length features selected then $\mathrm{x} 1$ will be grouped to the $\mathrm{x}$.

The algorithmic approach of the feature set optimization in pseudo code format

- For-each first order class label $\left.c l_{i}=\left\{k w g_{i}, a g_{i}, t z_{i}\right\} \forall i=1,2, \ldots \ldots .|T Z|\right\} / /$ since number 
of first order class labels are is equal to number of time zones

- Let $w s$ be the set of words opted after text preprocessing of the documents of $c l_{i}$.

- Let $c \omega_{\tau}$ be the concept weight threshold

- Order the terms belongs to $w s$ in descending by their frequency score.

- For each term $\{w \exists w \in w s\}$ Begin

- $c t \leftarrow w$ (add $w$ to $c t) / / c t$ is the concept tag to be formed and first $w$ is first word of the set ws

- For each term $\left\{w^{\prime} \mid w^{\prime} \in w s, w^{\prime} \neq w\right\}$ Begin

- $c t \leftarrow c t / /$ moving all elements of $c t$ to $c t^{\prime}$

- $c t^{\prime} \leftarrow w^{\prime} / /$ adding word $w^{\prime}$ to $c t^{\prime}$

- If $\left(c w(c t) \geq c \omega_{\tau}\right)$

- If $c w\left(c t^{\prime}\right) \cong c w(c t)$ then

- Discard $c t$;

- Set $c t \leftarrow c t$

- Else

- $c t s \leftarrow c t$

- Set $c t \leftarrow c t$

- End

- Else

- Discard $c_{i}^{\prime}$

- End;

- End

- Order $c t$ in descending order by the length of the concepts (here after concepts referred as features)

- Let $m l$ be the maximal length of the feature in $c t s$

- For each $\{c t \mid c t \in c t s ; t c(c t) \equiv m l\}$ move $c t$ to label set $l s$

- For each $\{l \exists l \in l s \wedge t c(l) \cong m l\} ;$ Here $t c(c)$ indicates the term count of the feature $c t$ Begin

- For each $\{c t \mid c t \in c t s ; c t \notin l s\} \quad$ Begin

- Find similarity score $s s_{(l \leftrightarrow c t)}=\frac{t c(l \cap c t)}{t c(l \bigcup c t)}$

- End

- For each $\{c t \exists c t \in c t s \wedge c t \notin l s\}$ Begin

- set $\bigcup_{j=1}^{|s|} s s_{\left(l_{j} \leftrightarrow c t\right)}$ in descending order and select first element as $s s_{l \leftrightarrow c t}$

- If $\left(s s_{l \leftrightarrow c t} \geq s s_{\tau}\right)$ then consider $c t$ as feature of the group represented by the label $l$

- Else move $c t$ to $l s$

- End;

- End

Return $l s$ as set of second order class labels

Further the classification of the documents is done that performs supervised learning by using concept labels as the labels of the categories

\subsection{Third Order Classification by Correlation of the Semantics as Features}

This stage of supervised learning estimates the correlation between activities that extracted from the given documents of each second order class label. In this regard the activities found are considered to be categorical as they associate with divergent arguments. Henceforth here we use mean-square contingency coefficient ${ }^{22}$ to estimate the correlation between attributes. Any given two activities $\mathrm{A}$ and $B$ such that $\left\{a_{1}, a_{2}, a_{3}, \ldots . . a_{m}\right\},\left\{b_{1}, b_{2}, b_{3}, \ldots . b_{n}\right\}$ are categorical arguments found to be associated to $\mathrm{A}$ and $\mathrm{B}$ respectively. The size of the set of arguments associated with activity $A$ is $m$ and activity $B$ is $n$. Then the mean square contingency coefficient between activities A and B can be measured as follows:

$$
\rho_{i j}=\sum_{i=1}^{m} \sum_{j=1}^{n} 1-\frac{1}{o\left(a_{i}, b_{j}\right)}
$$

Here in this equation $\rho_{i j}$ is the fraction of co-occurrence of $a_{i}, b_{j}$

$$
\rho_{i}=\sum_{i=1}^{m} 1-\frac{1}{o\left(a_{i}\right)}
$$

Here in this equation $\rho_{i}$ is the fraction of occurrence of $a_{i}$

$$
\rho_{j}=\sum_{j=1}^{n} 1-\frac{1}{o\left(b_{j}\right)}
$$

Here in this equation $\rho_{j}$ is the fraction of occurrence of $b_{j}$

$$
\chi_{(A \leftrightarrow B)}^{2}=\frac{1}{\min (m, n)-1} * \sum_{i=1}^{m} \sum_{j=1}^{n} \frac{\left(\rho_{i j}-\left(\rho_{i} \cdot \rho_{j}\right)\right)^{2}}{\rho_{i} \cdot \rho_{j}}
$$

Here in this equation $\chi_{(A \rightarrow B)}^{2}$ is the mean square contingency coefficient that indicates the correlation between activities $\mathrm{A}$ and $\mathrm{B}$.

According to the correlation estimation process explored here, the activities that are highly correlated will be grouped. Further each group of activities will be used as class label for second level of supervised learning.

\section{Empirical Study}

\subsection{Dataset Characteristics}

The features of the datasets used in experiments have an important role. A set of research articles published 
Table 1. Statistics of the experiment results

\begin{tabular}{|l|c|}
\hline Total Number of documents & $\mathbf{6 4 2 5}$ \\
\hline Approximate number of topics related to articles & 32 \\
\hline Average number of sub topics related to main topics & 4 \\
\hline Total number of time zones found & 31 \\
\hline Total number author groups found & 31 \\
\hline Total number of keyword groups found & 31 \\
\hline First order class labels count & 31 \\
\hline Second order class labels count & 94 \\
\hline Third order class labels count & 324 \\
\hline
\end{tabular}

in reputed international journals and conferences has been considered as input source for experiments. The research articles selection is done under certain criterions such as 1 . Should have metadata that reflects versioning dates, authors and keywords, 2. Articles of decent count (not less than 2\%) should available for each topic (generally reflects by the keywords) and sub topic (generally reflected by the terms found the article content) (Table 1).

\subsection{Performance Analysis}

Total number of documents considered 6425, total number of documents found to be classified to accurate class labels are 6009 and to false class labels are 416 .

As per the results explored in Table 1, the devised hierarchical supervised learning of documents is accurate to the level of $91.46 \%$. The failure percentage is $8.53 \%$, which is nominal.

The experiments also conducted on the same data set with earlier method called optimizing features by correlating $^{21}$ that is not considering the semantic similarities of the features and CSS-HDC 22 , which is considering the semantic relations but not considering the context labeling. The results observed are:

Total number of documents found to be classified by model devised in ${ }^{21}$ are
- to accurate class label are 4150

- $\quad$ and to false class label are 2284

As per these results, the accuracy of the model devised in $^{21}$ is limited to $64.5 \%$.

Total number of documents found to be classified by model devised in ${ }^{22}$ are

- to accurate class label are 5334

- and to false class label are 1091

As per these results, the accuracy of the model devised in ${ }^{22}$ is limited to $83 \%$.

Hence it is obvious to conclude that the context similarity along with concept and semantic similarity score is more significant to achieve classification accuracy towards the supervised learning (details explored in Table 2).

\section{Conclusion}

Here in this paper, a novel three level ordered supervised learning strategy has been devised. The said model classifies the documents by context labels in first order classification. These context labels are formed by using metadata of the documents. The second order classifier of the OCCSR classifies the documents of the each context label, which is done by concept labels. These concept labels are formed by estimating the correlation of the features observed. Further the documents of the each concept label are classified again by estimating the semantic similarities in third order classification of the OCCSR. The main contribution of this paper is defining class labels using document metadata to assess the context relevancy of the documents. This is further integrated with our earlier models concept labeling ${ }^{21}$, CSS-HDC ${ }^{22}$ to improve the sensitivity and accuracy of the supervised learning. The experimental results explored here are indicating the significance of the devised Document Classification by

Table 2. Classification statistics of the concept labeling ${ }^{21}$, CSS-HDC ${ }^{22}$ and OCCSR

\begin{tabular}{|l|c|c|c|c|c|}
\hline & \multicolumn{3}{|c|}{ No of Labels } & \multicolumn{2}{c|}{ Classification Accuracy } \\
\hline & $\begin{array}{c}\text { Context } \\
\text { Labeling }\end{array}$ & $\begin{array}{c}\text { Concept } \\
\text { Labeling }\end{array}$ & $\begin{array}{c}\text { Semantic } \\
\text { Labeling }\end{array}$ & $\begin{array}{c}\text { True Labeling } \\
\text { (in \%) }\end{array}$ & False Labeling \\
\hline Concept Labeling [21] & 0 & 91 & 0 & 65 & 35 \\
\hline CSS-HDC [22] & 0 & 91 & 257 & 83 & 17 \\
\hline OCCSR & 31 & 94 & 324 & 92 & 8 \\
\hline
\end{tabular}


the Order of Context, Concept and Semantic Relations is Scalable and Robust. In particular the model devised here is most useful to assess relation of the documents published in social communities like electronic journals, publishers and blogs. The future research can aim to apply this ordered classification on search logs to refine the search results recommendation.

\section{References}

1. Aas K, Eikvil L. Text categorisation: A survey. Report No: 941; 1999 Jun.

2. Salton G, Wong A, Yang CS. A vector space model for automatic indexing. Communications of the ACM. 1975 Nov; 18(11):613-20.

3. Salton G . Introduction to Modern Information Retrieval. NewYork: McG raw-Hill; 1983.

4. Huang A. Similarity measures for text document clustering. Proceedings of the 6th New Zealand Computer Science Research Student Conference (NZCSRSC'2008); Christchurch, New Zealand. 2008 Apr. p. 49-56.

5. Debole F, Sebastiani F. Supervised term weighting for automated text categorization. Text mining and its applications. Berlin, Heidelberg: Springer; 2004; 138:81-97.

6. Clinchant S, Gaussier E. Information-based models for ad hoc IR. Proceedings of the 33rd international ACM SIGIR Conference on Research and Development in Information Retrieval. ACM; 2010 Jul. p. 234-41.

7. Sebastiani F. Machine learning in automated text categorization. ACM Computing Surveys (CSUR). 2002 Mar; 34(1):1-47.

8. Taira H. Text categorization using machine learning Doctoral [Dissertation]. Nara Institute of Science and Technology; 2002.

9. Vapnik VN, Vapnik V. Statistical Learning Theory. New York: Wiley; 1998.

10. Dumais S, Platt J, Heckerman D, Sahami M. Inductive learning algorithms and representations for text categorization. Proceedings of the 7th International Conference on Information and Knowledge Management; 1998 Nov. p. 148-55.

11. Yang Y, Liu X. A re-examination of text categorization methods. Proceedings of the 22nd Annual International
ACM SIGIR Conference on Research and Development in Information Retrieval; 1999 Aug. p. 42-9.

12 Guan Y. Methods for pattern classification. INTECH Open Access Publisher; 2010.

13. Cooley R. Classification of news stories using support vector machines. Proceedings of 16th International Joint Conference on Artificial Intelligence Text Mining Workshop; 1999 Aug.

14. Ozgur A, Gungor T. Classification of skewed and homogenous document corpora with class-based and corpus-based keywords. Advances in Artificial Intelligence (KI 2006). Berlin, Heidelberg: Springer; 2007. p. 91-101.

15. Yang Y, Pedersen JO. A comparative study on feature selection in text categorization. ICML; 1997 Jul. p. 412-20.

16. Debole F, Sebastiani F. Supervised term weighting for automated text categorization. Text mining and its applications. Berlin, Heidelberg: Springer; 2001. p. 81-97.

17. Ozgur A, Ozgur L, Gungor T. Text categorization with class-based and corpus-based keyword selection. Computer and Information Sciences-ISCIS 2005. Berlin, Heidelberg: Springer; 2005. p. 606-15.

18. Tasci S, Gungor T. An evaluation of existing and new feature selection metrics in text categorization. 23rd International Symposium on Computer and Information Sciences (ISCIS’08); 2008 Oct. p. 1-6.

19. Tasci S, Gungor T. Comparison of text feature selection policies and using an adaptive framework. Expert Systems with Applications. 2013 Sep; 40(12):4871-86.

20. Liu Y, Loh HT, Sun A. Imbalanced text classification: A term weighting approach. Expert systems with Applications. 2009 Jan; 36(1):690-701.

21. Venkata Ramana A, Naidu MM. Optimizing features by correlating for concept labeling in text classification. 2014 IEEE International in Advance Computing Conference (IACC). Gurgaon: IEEE; 2014 Feb 21-22. p. 561-7.

22. Venkata Ramana A, Kesavulu Reddy E. Hierarchical document classification by conceptual and semantic similarities. IJSAIT. 2014 Sep; 3(4):12-7.

23. Jayaraj V, Mahalakshmi V. Augmenting efficiency of recruitment process using IRCF text mining algorithm. Indian Journal of Science and Technology. 2015 Jul; 8(16):53381. 